Planta (Berl.) 119, 351-360 (1974)

(C) by

\title{
Measurement of Oxygen Partial Pressure within Soybean Nodules by Oxygen Microelectrodes
}

\author{
J. D. Tjepkema and C. S. Yocum* \\ Department of Botany, University of Michigan, \\ Ann Arbor, Michigan 48104, USA \\ Received May 21/July 10, 1974
}

Summary. The internal $\mathrm{pO}_{2}$ of soybean (Glycine max Merr.) nodules was measured with oxygen microelectrodes. For nodules in air at $23^{\circ}$, the $\mathrm{pO}_{2}$ decreased sharply across the nodule cortex, and was too low to measure in the central tissue. At $1^{\circ}$ in $1.0 \mathrm{~atm} \mathrm{O}_{2}$, the $\mathrm{pO}_{2}$ in the central tissue was measurable, and was approximately uniform from the center to the edge of the central tissue. This uniformity was probably due to the intercellular air spaces of the central tissue, since infiltrating the spaces with water substantially decreased the $\mathrm{pO}_{2}$ in the central tissue. The results strongly suggest that most of the resistance to $\mathrm{O}_{2}$ diffusion into the nodule occurs within the cortex.

\section{Introduction}

It appears that the rate of respiration by the bacteroids in soybean nodules is limited by the rate of oxygen diffusion into the nodule, and we have argued that the nodule cortex is the source of most of this resistance (Tjepkema, 1971; Tjepkema and Yocum, 1973). Bergersen and Goodchild (1973) have disagreed with this suggestion, but the following results with oxygen microelectrodes strongly support our hypothesis.

\section{Materials and Methods}

Two different sizes of electrodes, and several electrodes of each size were used. The larger size of microelectrode was made by sharpening 26 -gauge platinum wire by electrocorrosion, coating with a low-melting-point glass (Wolbarsht et al., 1960), and exposing the platinum by cutting off the glass tip with a razor blade. This resulted in a roughly cylindrical area of exposed platinum at the electrode tip, with a diameter of $40-60 \mu \mathrm{m}$ and a length of $30-60 \mu \mathrm{m}$.

The smaller size of microelectrode was purchased from the Transidyne General Corp. (Ann Arbor, Mich., USA) and was model No. 721. These were glass-insulated platinum with a tip diameter of $2 \mu \mathrm{m}$ and a $10-\mu \mathrm{m}$ length of exposed platinum.

\footnotetext{
* To whom reprint requests should be sent.
} 


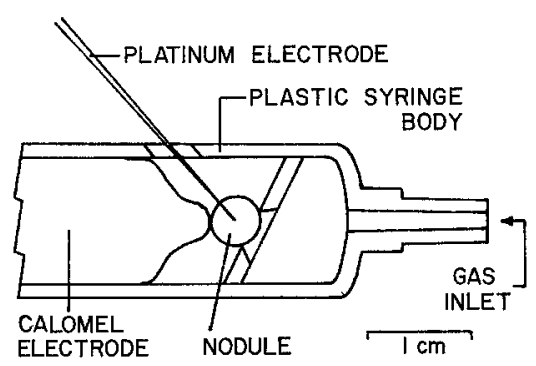

Fig. 1. Chamber for oxygen microelectrode measurements

Soybeans, Glycine max Merr. ev. Chippewa (Ypsilanti Farm Bureau, Ypsilanti, Mich., USA), were grown as described previously (Tjepkema and Yocum, 1973). The nodules were held in place by the arrangement shown in Fig. 1. A mechanical drive was used to advance the electrodes into the nodules. For the 50- $\mu \mathrm{m}$ tip electrodes, the current was measured with a Keithly model 150 A microvolt-amperometer (Keithley Instruments, Cleveland, Ohio, USA), and the apparatus was enclosed in a Faraday cage made of copper screen. The microelectrodes were maintained at $-0.64 \mathrm{~V}$ with respect to the calomel reference electrode. For the $2-\mu \mathrm{m}$ tip electrodes, the current was measured with a Keithley model $610 \mathrm{~B}$ electrometer, and the apparatus was enclosed in an aluminium box. The microelectrodes were maintained at $-0.51 \mathrm{~V}$ with respect to the reference electrode. Measurements were made after the current reached an approximately steady state. This was about $40 \mathrm{~s}$ for the $50-\mu \mathrm{m}$ tip electrodes and less than $10 \mathrm{~s}$ for the $2-\mu \mathrm{m}$ tip electrodes.

Before and after each use the electrodes were calibrated in an air-equilibrated agar gel, containing $0.1 \mathrm{M} \mathrm{KCl}$. The relationship between the electrode current in tissue and the current in $0.1 \mathrm{M} \mathrm{KCl}$ is unclear, especially due to the leghemoglobin and intercellular air spaces in the nodule. These are unevenly distributed in the nodule and their effect on the oxygen-electrode ourrent cannot be accurately calculated. Thus the electrode current must be considered only qualitatively related to the $\mathrm{pO}_{2}$ in the nodule.

In preliminary vacuum infiltration experiments (see Discussion), India ink No. 4415 from Higgins Ink Co., Newark, N. J., USA, and Triton X-100 detergent from Sigma Chemical Co., St. Louis, Mo., USA, were used.

\section{Results}

The 50- $\mathrm{m}$ tip electrodes were used for the experiments of Figs. 2-4. Fig. 2 shows a typical set of measurements at $23^{\circ}$. The measurements in helium and air were taken along adjacent but nonintersecting paths in the same nodule. With air in the electrode chamber, the current decreased rapidly as the electrode was advanced into the nodule, and then reached an approximately steady level as the electrode crossed most of the nodule interior. As the electrode approached the exterior on the opposite side of the nodule, the current increased sharply.

Measurements were not taken completely to the opposite nodule surface, because of the dànger of damaging the electrode by driving it 


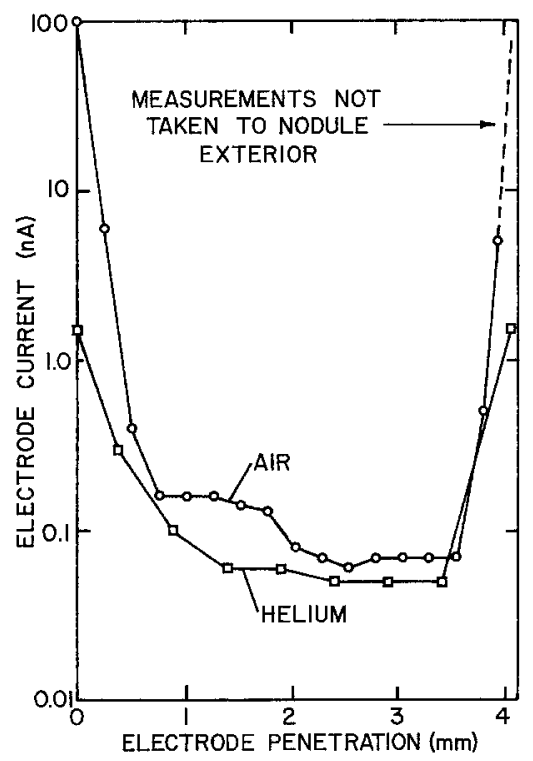

Fig. 2. Oxygen microelectrode current as the electrode is advanced through a nodule at $23^{\circ}$. Electrode calibration $(0.1 \mathrm{M} \mathrm{KCl}): 43 \mathrm{nA}$ before measurements, $52 \mathrm{nA}$ afterwards

into the plastic holding the nodule in place. In helium, the electrode current near the nodule exterior was much lower than it was in air. In the nodule center, the electrode current in helium was sometimes lower than in air, as shown in Fig. 2. But in a similar number of other determinations the situation was the reverse, indicating that these differences were not significant. This electrode current in the nodule center was probably due to reduction of substances other than oxygen at the electrode surface. When the electrode was withdrawn from the nodule and was no longer touching it, there was a small negative current, ranging from almost zero to a magnitude almost as large as observed in the nodule center, but of negative sign. This was probably a stray current, due to imperfect electrostatic shielding. Note that in helium the electrode current increased near the nodule exterior. This was probably because the helium flow rate was not sufficient to exclude all $\mathrm{O}_{2}$ from the measuring chamber. In any event, this current was only about $3 \%$ of the current in air at the nodule exterior.

In the results of Fig. 2 , the internal $\mathrm{pO}_{2}$ of the nodule was measurable only near its exterior. To increase the $\mathrm{pO}_{2}$ to measurable levels, we made measurements in 1.0 atm $\mathrm{O}_{2}$, lowered the temperature to $1^{\circ}$, 


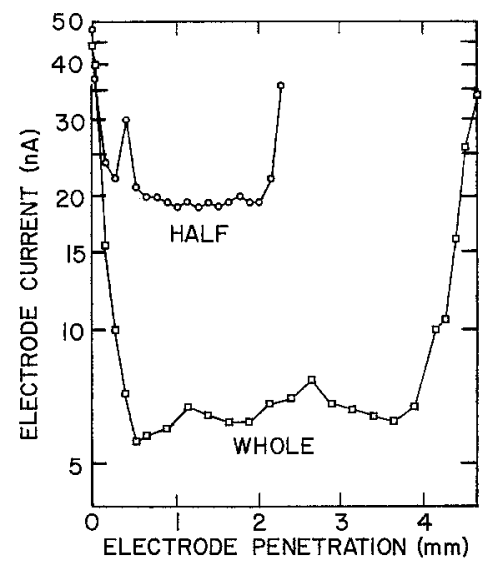

Fig. 3. Oxygen microelectrode current before and after cutting a nodule in half. The measurements were made in 1.0 atm $\mathrm{O}_{2}$ at $1^{\circ}$. Electrode calibration $(0.1 \mathrm{M} \mathrm{KCl})$ : $12 \mathrm{nA}$ before and after measurements

and cut the nodule in half. Fig. 3 shows measurements before and after cutting a nodule in half. The electrode current was constant in the nodule interior, and increased near the nodule exterior and the cut surface. Cutting the nodule in half substantially increased the internal $\mathrm{pO}_{2}$.

The uniformity of central tissue $\mathrm{pO}_{2}$ shown in Fig. 3 was probably due to the well-developed intercellular air spaces of the central tissue. To determine whether this was so, we measured the $\mathrm{pO}_{2}$ before and after infiltrating the spaces of the nodule with water. Infiltration was done by placing a half-nodule in water under a vacuum, releasing the vacuum, and repeating this procedure 2 more times in a 5 -min period. The effectiveness of this procedure was checked by repeating it with a saturated aqueous solution of aniline blue black. Dye was found in at least twothirds of the central tissue volume. If whole rather than half-nodules were used, no dye was found in the central tissue. Thus the dye must have entered the half-nodule via the cut surface of the nodule. Fig. 4 gives typical electrode measurements for nodules before and after infiltration with water. In larger nodules, infiltrating the air spaces with water greatly decreased the internal $\mathrm{pO}_{2}$ so that in the center of the nodule, the electrode current was about the same as the residual electrode current measured in helium. In smaller nodules the effect was less, but in all cases, after water infiltration, the internal $\mathrm{pO}_{2}$ of the nodule was reduced and was no longer uniform in the center of the nodule. The reason why the effect of infiltration was so much less in smaller nodules is not clear. 


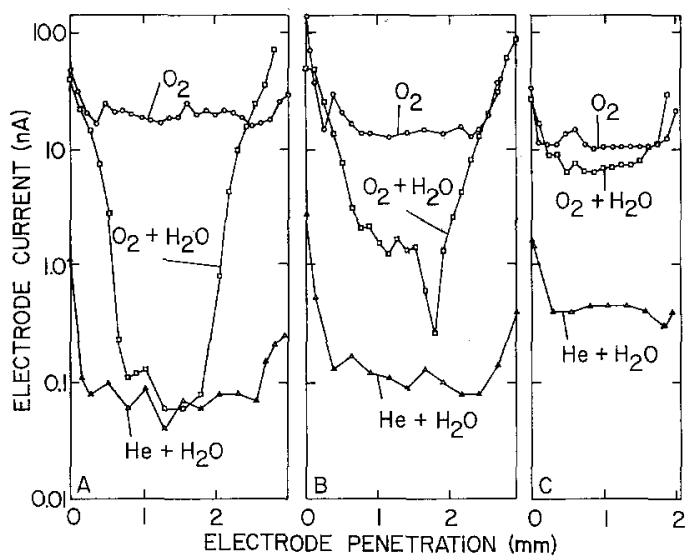

Fig. 4A-C. Oxygen microelectrode current in half nodules, before and after infiltrating the intercellular air spaces with water. The measurements were made in $1.0 \mathrm{~atm} \mathrm{O}_{2}$ at $1^{\circ}$. Electrode calibration $(0.1 \mathrm{M} \mathrm{KCl})$ before and after measurements: (A) 10 and $13 \mathrm{nA}$; (B) 25 and $19 \mathrm{nA}$; (C) 9 and $20 \mathrm{nA}$, respectively

Figs. 5-7 show results with the $2-\mu$ m electrodes. These measurements were taken at $23^{\circ}$. In Fig. 5 the electrode penetrated the cortex at a point between the vascular strands, while in Fig. 6 the electrode penetrated through a vascular strand. The cortex was substantially thicker near the vascular strands, because of ridges on the nodule cortex above the strands and extension of the cortical tissue into the central tissue below the strands. After the measurements were made, the electrode was advanced about $1 \mathrm{~mm}$ further into the nodule, so that the hole made by the electrode was enlarged, and the path of electrode though the nodule could be more easily traced. The nodule was then cut in half under a stereomicroscope, so that the razor blade cut through the hole made by the electrode, and a free-hand section from the cut surface was examined under the microscope. Figs. 5 and 6 were chosen from 22 such measurements, because they were among the few in which the exact, path of the electrode was found. It should be noted that the electrodes often broke during measurements; such measurements were discarded.

When a measurement was taken between the vascular strands, as exemplified by Fig. 5, the electrode current in the outer part of the cortex was quite irregular but was usually at least as large as the current when the electrode was inserted into agar equilibrated with air. There were often one or more large current peaks as the electrode was advanced into the cortex. These were up to 10 times larger than the electrode current in agar, and occurred at variable distances from the nodule exterior. 


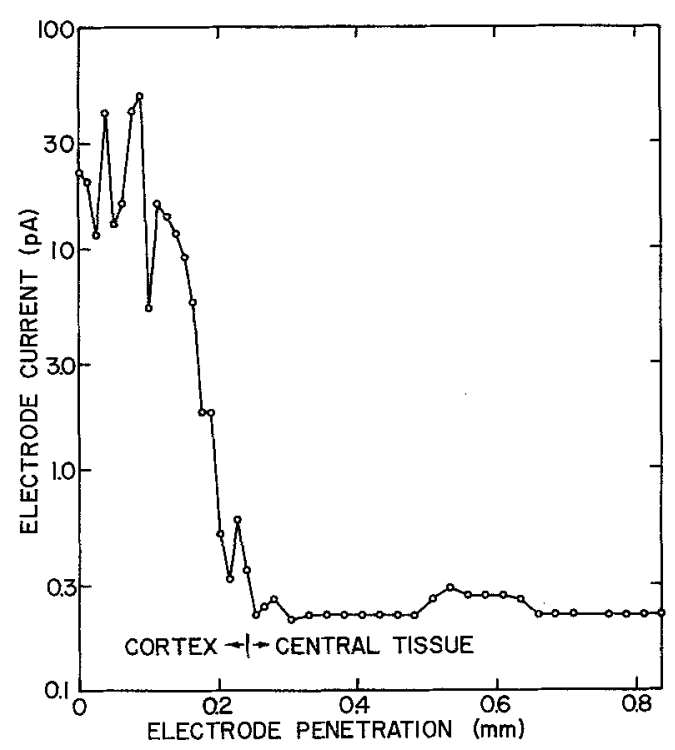

Fig. 5. Oxygen microelectrode current as the electrode is advanced through the cortex between vascular strands. Temperature: $23^{\circ}$. Electrode calibration (0.1 M $\mathrm{KCl})$ : $14 \mathrm{pA}$ before measurements, $26 \mathrm{pA}$ afterwards

These peaks were due to $\mathrm{O}_{2}$, since flushing the electrode chamber with $\mathrm{N}_{2}$ reduced the current to a very low value. The cause of the peaks was probably contact of the electrode with the large intercellular air spaces which are present in some parts of the outer cortex. In the inner part of the cortex, the electrode current decreased sharply to a very low value, and all or almost all of this decrease occurred before the electrode entered the central tissue. In the central tissue, the electrode current was relatively constant as the electrode was advanced and did not decrease when $\mathrm{N}_{2}$ was flushed through the electrode chamber. This current may have partly been a residual current as discussed with regard to the $50-\mu \mathrm{m}$ tip electrodes, but was partly due to other causes. When the oxygen electrode was in place but not touching the nodule, the current was $0.14 \mathrm{pA}$. This may have been a stray current as discussed previously, or since it was of positive sign, it may have been due to current leakage around the electrodes. This difference in results between the $2-\mu \mathrm{m}$ and $50-\mu \mathrm{m}$ tip electrodes was not surprising since the apparatus used was not exactly the same, especially with regard to electrostatic shielding.

Fig. 6 was the only result obtained in which it was clear that the electrode passed through the center of a vascular strand when going through the cortex. The electrode current fell sharply as the electrode 


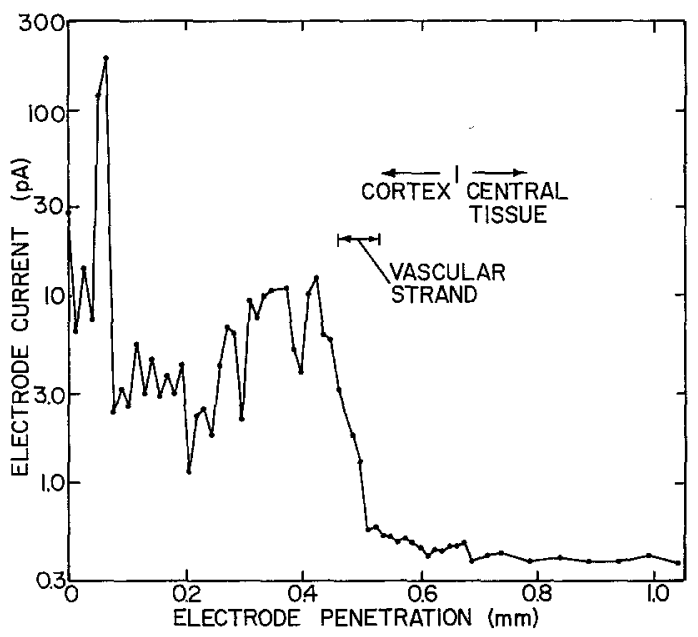

Fig. 6. Oxygen microelectrode current as the electrode is advanced through the cortex, penetrating a vascular strand. Temperature: $23^{\circ}$. Electrode calibration

$(0.1 \mathrm{M} \mathrm{KCl}): 14 \mathrm{pA}$ before measurements, $17 \mathrm{pA}$ afterwards

penetrated into and through the vascular strands. In the area of the cortex between the strand and the central tissue there was no further decrease in the electrode current, and the current also remained constant as the electrode was advanced into the central tissue. In other results, in which it was not known if the vascular strand was penetrated, the main decrease in the electrode current sometimes occurred closer to the boundary of cortex and central tissue. In the part of the cortex above the vascular strands, irregular, large current peaks were often observed, as shown in the result of Fig. 6.

When the nodule of Fig. 6 was cut in half, an irregular cavity in its center was found. Fig. 7 shows the results when an oxygen microelectrode was advanced through the cut surface of the central tissue. The electrode current was much larger than was found in the central tissue of this same nodule in Fig. 6. This increased current was due to $\mathrm{O}_{2}$, since when nitrogen was flushed through the electrode chamber, the current decreased to a low value. Cavities occur only in about one of 100 very large nodules. When large nodules without cavities were cut in half, no increase in the electrode current in the central tissue was observed.

\section{Discussion}

First let us consider the spatial resolution of the oxygen electrodes. Davies (1962) calculated that at a radius of 10 times the electrode 


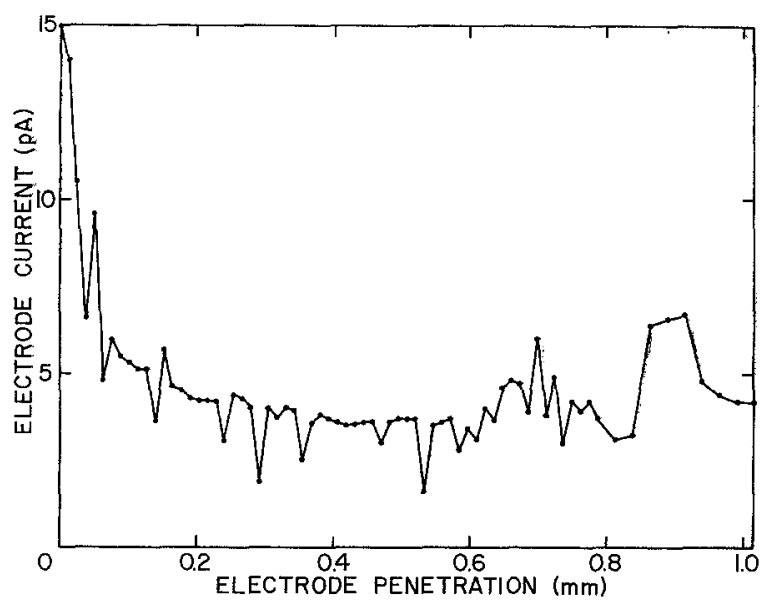

Fig. 7. Oxygen microelectrode current as the electrode is advanced into the central tissue of a half nodule which has a central cavity. Temperature: $23^{\circ}$. Electrode calibration $(0.1 \mathrm{M} \mathrm{KCl}): 17 \mathrm{pA}$ before measurements, $14 \mathrm{pA}$ afterwards

radius, the $\mathrm{O}_{2}$ concentration is at $90 \%$ of the concentration that would occur if no electrode were present. Thus the electrode measures the average $\mathrm{O}_{2}$ concentration in a sphere of about 10 times its radius. For the $50-\mu \mathrm{m}$ tip electrodes that we used, the average tip radius was about $25 \mu \mathrm{m}$, so they resolved a sphere of about $250 \mu \mathrm{m}$ radius. The $2-\mu \mathrm{m}$ tip electrodes had an average radius of about $3 \mu \mathrm{m}$, so they resolved a sphere of about $30 \mu \mathrm{m}$ radius. Thus the calculated resolution of the electrodes was adequate for these experiments. The actual resolution may have been better than calculated since our electrodes were aged, and the electrode current was several times lower than in new electrodes the platinum surfaces of which were more fully activated.

Now let us consider the implication of our results for the diffusion of oxygen through the cortex of the soybean nodule. Both Sprent (1972) and Bergersen and Goodchild (1973) have concluded that intercellular air spaces pass through the nodule cortex and form continuous gas passageways between the soil atmosphere and the intercellular air spaces of the central tissue. Tjepkema and Yocum (1973) came to a different conclusion and suggested that there may be a layer of the cortex through which no intercellular air spaces penetrate, and that most of the $\mathrm{pO}_{2}$ gradient between the external atmosphere and the bacteroids occurs at this layer. The results of Figs. 5 and 6 confirm this suggestion. Most of the $\mathrm{pO}_{2}$ gradient in these nodules occurred in the inner part of the cortex. Such a large $\mathrm{pO}_{2}$ gradient could occur only if very few or no intercellular air spaces penetrate this layer of tissue. 


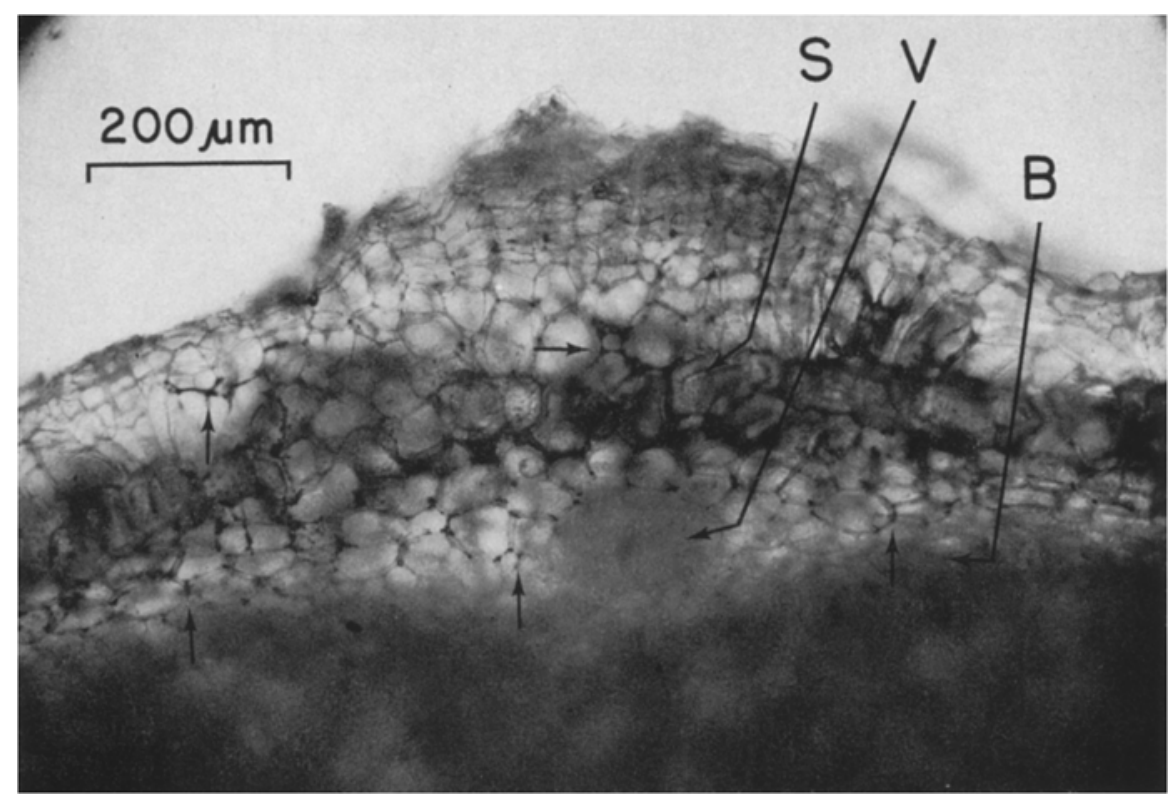

Fig. 8. Free-hand section of a nodule that has been vacuum infiltrated with India ink. The unlabeled arrows indicate intercellular spaces filled with ink. $V$, vascular strand; $S$, sclereid layer; $B$, boundary of cortex and central tissue. $\times 103$

Fig. 7 shows that when $\mathrm{O}_{2}$ diffusion can bypass the cortex, the $\mathrm{pO}_{2}$ in the central tissue can reach a much higher value. Apparently the intercellular air spaces of the central tissue communicate directly with the central cavity, so that when the cavity is exposed by cutting the nodule in half, the $\mathrm{pO}_{2}$ in the air spaces approaches the atmospheric value. When there is no cavity, cutting the nodule in half does not increase the $\mathrm{pO}_{2}$, probably because the intercellular air spaces of the central tissue are blocked at the cut surface. Such blockage seems reasonable because the walls of the cut-open cells have little rigidity, and the cutting might disrupt any hydrophobic coating on the air spaces which might function to prevent entry of water into the air spaces.

The 2- $\mu \mathrm{m}$ tips of the smaller electrodes used were about the same size as the intercellular air spaces of the central tissue. Thus if the tips were in or adjacent to the air spaces, one would expect the electrode current to be influenced by the $\mathrm{pO}_{2}$ in the air spaces. If this $\mathrm{pO}_{2}$ were a substantial fraction of the atmospheric value, one would expect the electrode current to be a substantial fraction of the current in air-equilibrated agar. As the electrodes were advanced through the central tissue (Figs. 5, 6) only very low current values were found. This was also true for all 
other measurements that we made in the central tissue. Thus it appears that the $\mathrm{pO}_{2}$ in the intercellular air spaces of the central tissue is quite low. If so, these spaces must not be directly connected with the external atmosphere.

The above results (Figs. 2-7) indicate that the inner cortex of the nodule may contain a barrier to oxygen diffusion into the central tissue. This was confirmed by preliminary experiments in which nodules were vacuum-infiltrated with India ink, by a modification of the method of Hinchman (1972). India ink was passed through a filter of $0.45 \mu$ pore size. The filtrate was vacuum evaporated to half of its original volume, and $0.15 \mathrm{ml}$ of a $10 \%$ aqueous solution of Triton X-100 detergent was added to the $1.5 \mathrm{ml}$ of concentrate. Fig. 8 shows a typical result. The ink penetrated the cortex to a depth of two or three cell layers inside of the layer of sclereid cells, but was never observed in the central tissue. This same result was found with a variety of dyes dissolved in ethanol or water, except the dyes rapidly diffused out of the intercellular spaces.

We also repeated the experiment of Bergersen and Goodchild (1973) who described a method which permits direct observation of gas in the intercellular air spaces of slices of soybean nodules. Our observations confirmed their results, except that we could find no air spaces that were continuous through the inner layer of the cortex, where the India ink did not penetrate in Fig. 8 .

From the above results and discussion we conclude, at least for soybean nodules as we have grown them, that the intercellular air spaces of the central tissue are functional, but are not connected by a gaseous pathway to the external atmosphere. We also conclude that most of the gradient in $\mathrm{pO}_{2}$ between the external atmosphere and the bacteroids occurs in the inner layer of the cortex of the nodule.

\section{References}

Bergersen, F. J., Goodchild, D. J.: Aeration pathways in soybean root nodules. Aust. J. biol. Sci. 26, 729-740 (1973)

Davies, P. J.: The oxygen cathode. In: Physical techniques in biological research, vol. IV, p. 137-179, Nastuk, W. L., ed. New York-London: Acad. Press 1962

Hinchman, R. R.: The intercellular air spaces in oat seedlings: an interconnected tubular system. Argonne Nat. Lab. (Argonne, Ill., USA), Biol. and Med. Res. Ann. Rept. 1972, pp. 115-116

Sprent, J. I.: The effects of water stress on nitrogen-fixing root nodules. II. Effects on the fine structure of detached soybean nodules. New Phytol. 71, 443-450 (1972)

Tjepkema, J. D.: Oxygen transport in the soybean nodule and the function of leghemoglobin. Doct. dissert., Univ. of Michigan, Ann Arbor 1971

Tjepkema, J. D., Yocum, C. S.: Respiration and oxygen transport in soybean nodules. Planta (Berl.) 115, 59-72 (1973)

Wolbarsht, M. L., MacNichol, E. F., Wagner, H. G.: Glass insulated platinum microelectrode. Science 132, 1309-1310 (1960) 